DOI: 10.12957/demetra.2017.26371

\title{
Política Nacional de Alimentação e Nutrição: avaliação da implantação de programas em municípios baianos
}

\section{National Food and Nutrition policy: evaluating the implementation of programs in municipalities of the state of Bahia, Brazil}

Lana Mércia Santiago de Souza ${ }^{1}$

Sandra Maria Chaves dos Santos²

${ }^{1}$ Universidade Federal da Bahia, Escola de Nutrição, Núcleo de Nutrição e Políiticas Públicas. Salvador-BA, Brasil.

2 Universidade Federal da Bahia, Departamento de Ciências da Nutrição, Pós-graduação em Alimentos, Nutrição e Saúde. Salvador-BA, Brasil.

Financiamento: projeto financiado pela Fundação de Amparo a Pesquisa do Estado da Bahia. Edital PPSUS 020/2013.

Correspondência / Correspondence Lana Mércia Santiago de Souza

E-mail: lanamercia@gmail.com

\section{Resumo}

Introdução: A Política Nacional de Alimentação e Nutrição (PNAN) propõe uma atuação de caráter interdisciplinar e intersetorial, com o intuito de integrar as diversas ações para enfrentamento dos agravos nutricionais, organizado por uma série de programas e ações em diferentes vertentes, a serem implementados de maneira descentralizada, junto ao Sistema Único de Saúde. Objetivo: avaliar a implantação de programas da PNAN em municípios baianos e relacioná-los a indicadores sociodemográficos. Método: pesquisa documental retrospectiva, entrevistas semiestruturadas com informantes-chave e questionário estruturado on-line com gestores locais, via plataforma Survey Monkey. As ações do âmbito municipal foram então avaliadas quanto ao grau de implantação e os resultados foram associados com indicadores sociodemográficos, com uso de testes estatísticos. Resultados e discussão: A caracterização da PNAN e suas ações revelaram o quanto a política é ampla e dinâmica e por isso também complexa e de difícil implantação. Os programas de maior tempo de existência apresentaram maior porcentual de implantação satisfatória nos municípios com relação às ações/programas mais recentes. Os indicadores de desenvolvimento humano, cobertura da Estratégia de Saúde da Família e de Insegurança Alimentar apresentaram associação estatisticamente significante com a existência de programas da política. Conclusões: Pelos resultados obtidos neste estudo, e considerando os municípios pesquisados, permanecem sendo priorizadas na implantação da PNAN ações de controle dos agravos nutricionais, como deficiência de ferro 
e de vitamina A, em detrimento de ações de promoção da alimentação saudável e prevenção de problemas nutricionais.

Palavras-chave: Política Nutricional. Descentralização. Saúde Pública. Promoção da Saúde.

\section{Abstract}

Introduction: The National Foodand Nutrition Policy (PNAN) proposes an interdisciplinary and intersectoral approach of operation in order to integrate several actions that address nutritional problems. PNAN is organized by a set of programs and actions in different areas to be implemented in a decentralized manner by the National Health System (SUS). Objective: To evaluate the implementation of PNAN programs in municipalities of the state of Bahia (brazil) and associate them with sociodemographic indicators. Method: This study was conducted by three strategies: retrospective documentary research, semistructured interviews with key informants and online structured questionnaire with local managers via the Survey Monkey platform. PNAN programs in the municipalities were statistically assessed for level of implementation and associated with sociodemographic indicators. Results and discussion: It was observed that although PNAN actions are broad and dynamics, they are still complex and difficult to implement. Long-term programs had higher percentage of satisfactory implementation when compared to short-term programs. The indicators for human development, coverage of the Family Health Strategy and Food Insecurity had a statistically significant association with the existence of political programs. Conclusions: In the municipalities in this study, implementation of PNAN continued to prioritize actions for control of nutritional problems, e.g. iron and vitamin A deficiency, to the detriment of actions for promotion of healthy eating and prevention of nutritional problems.

Keywords: Nutrition Policy. Decentralization. Public Health. Health Promotion. 


\section{Introdução}

A Política Nacional de Alimentação e Nutrição (PNAN), implantada em 1999 e atualizada em 2011, representa a proposição de uma política de caráter interdisciplinar e intersetorial, que tem como objetivo integrar as diversas ações para o enfrentamento dos agravos nutricionais, como parte da Política Nacional de Saúde. Insere-se, ao mesmo tempo, no contexto da Segurança Alimentar e Nutricional e do Direito Humano à Alimentação Adequada. ${ }^{1-3}$

Trata-se, assim, de uma proposta pioneira no setor, que propõe lidar com um cenário complexo de perfil nutricional, no qual coexistem quadros de desnutrição, deficiência de micronutrientes, excesso de peso e doenças crônicas não transmissíveis nas mesmas comunidades e, muitas vezes, no mesmo domicílio. ${ }^{4,5}$ Desse modo, propõe uma série de programas e ações em diferentes vertentes, que vão da promoção da saúde ao cuidado integral aos agravos nutricionais instalados.

Essas ações e programas devem ser organizados de maneira descentralizada, junto ao Sistema Único de Saúde (SUS), tendo a atenção básica à saúde como espaço privilegiado para o seu desenvolvimento. Com isso, pressupõe-se um cuidado longitudinal e territorial que leva em conta os determinantes sociais envolvidos no processo saúde-doença. Nesse contexto, é responsabilidade das três esferas de gestão - federal, estadual e municipal - a implementação da política, contudo, é no contexto local que a proposta se efetiva.

No entanto, o processo de descentralização de políticas não é linear e apresenta um conjunto de elementos como atores, interesses, recursos, estrutura, entre outros que, por pressuposto, modificam as políticas públicas. ${ }^{6}$ Além do mais, a cultura avaliativa de ações públicas e sua institucionalidade no Brasil ainda são incipientes, o que dificulta uma leitura mais aproximada do desenrolar das ações nas capilaridades federativas.

Um balanço macroestrutural das ações da PNAN aponta avanços e desafios a sua efetivação. Entre os avanços, cita-se a configuração e o desenvolvimento da vigilância alimentar e nutricional; a produção regular de informações sobre estado nutricional, por meio de pesquisas de base populacional; a construção da agenda de promoção da alimentação saudável; e a capacitação de recursos humanos. Podem ser destacadas como principais desafios a institucionalidade da área, a organização do processo de trabalho, o financiamento e o controle social. ${ }^{7}$

Assim, cabe conhecer, de fato, quais dessas inúmeras iniciativas estão implantadas em nível local e qual o seu grau de implantação, a fim de compreender como a PNAN está desenhada nesses espaços, reconhecendo seus alcances e limitações. Objetiva-se, destarte, avaliar a implantação de programas da PNAN em municípios baianos segundo grau de implantação e relacionando-os com indicadores sociodemográficos. 


\section{Metodologia}

Trata-se de um estudo qualitativo-quantitativo, realizado em municípios da Bahia. O estado é composto de 417 municípios, a maioria de pequeno porte populacional, tendo como capital a cidade de Salvador. ${ }^{8}$ Na Pesquisa Nacional por Amostra de Domicílios (2009), todos os estados do Nordeste registraram proporções inferiores à registrada para a média nacional $(69,8 \%)$ de Segurança Alimentar e Nutricional. O Estado da Bahia apresentou 58\% de segurança alimentar, estando os $42 \%$ restantes em algum nível de insegurança alimentar. ${ }^{9} \mathrm{O}$ Índice de Desenvolvimento Humano (IDH) é de 0,660, considerado médio, classificando-se na 22ª posição no país. ${ }^{10}$

A metodologia foi dividida em três momentos. O Momento 1 teve como foco a investigação dos programas que integram a PNAN na esfera da União, conforme previsto nos documentos da política. Utilizou-se como método a pesquisa documental retrospectiva em páginas on-line e em documentos institucionais do Ministério da Saúde, contemplados no período de vigência da PNAN e no período do estudo (1999-2015). A pesquisa foi realizada entre setembro de 2014 e setembro de 2015 .

O Momento 2 caracterizou a atuação da esfera estadual na gestão da política. Realizou-se uma entrevista semiestruturada com informante-chave atuante na área técnica de alimentação e nutrição, na Secretaria Estadual de Saúde. O Momento 3 identificou as ações e os programas implantados nos municípios baianos, por meio de um questionário estruturado on-line, disponibilizado através da plataforma Survey Monkey, e entrevistas semiestruturadas com informantes-chave (gestor ou responsável técnico) de municípios beneficiados com o Fundo Nacional de Saúde para Ações de Alimentação e Nutrição (FAN).

O universo do estudo foi composto inicialmente pelos 417 municípios baianos, que foram contatados por meio eletrônico. Desses, um total de 55 responderam ao questionário. Todos os participantes aceitaram compor a pesquisa, através da assinatura ou adesão on-line ao termo de consentimento livre e esclarecido. Esta pesquisa recebeu parecer favorável do Comitê de Ética da Escola de Nutrição da Universidade Federal da Bahia, parecer no 1.231.580, de 2015.

Cada programa/ação foi investigado a partir das seguintes variáveis: presença/ausência dos programas, financiamento local das ações, local de realização (se realizado em todas as unidades de saúde), prática de planejamento e avaliação das ações. Para avaliar o nível de implantação dos programas, propôs-se uma classificação, de modo que cada variável foi pontuada com 2 pontos, somando-se 10 pontos possíveis, de forma a obter os seguintes resultados: Não implantado, 0 (zero) ponto; Implantação insuficiente, de 2 a 4 pontos; Implantação satisfatória, de 6 a 8 pontos; e Implantação completa, 10 pontos. O resultado obtido refere-se ao grau de implantação de cada programa por município. 
No segundo momento, essas variáveis foram relacionadas aos indicadores de porte populacional, Índice de Desenvolvimento Humano Municipal (IDHM), situação de Segurança Alimentar e Nutricional (SAN), cobertura da Estratégia de Saúde da Família (ESF) e estado nutricional de crianças menores de 5 anos. ${ }^{11}$

O porte populacional é um dos indicadores aplicados em estudos de avaliação na área de saúde, associado a ações realizadas na atenção básica. ${ }^{12,13}$ Para classificação segundo porte populacional, os municípios foram organizados com base no modelo apresentado pelo Instituto Brasileiro de Geografia e Estatística ${ }^{14}$ (Quadro 1) e dicotomizados para análise de associação.

Quadro 1. Indicadores e categorias de classificação.

\begin{tabular}{|c|c|}
\hline Indicadores & Categorias \\
\hline Porte Populacional & Muito pequeno: população até 20.000 habitantes \\
& $\begin{array}{c}\text { Pequeno: população de } 20.001 \text { a } 50.000 \text { habitantes } \\
\text { Médio: população de } 50.001 \text { a } 100.000 \text { habitantes } \\
\text { Grande: população com mais de } 100.000 \text { habitantes }\end{array}$ \\
\hline IDHM & Baixo: 0,00 a 0,49 \\
Médio: 0,50 a 0,79 \\
Alto: 0,80 a 1,0 \\
\hline de desempenho do & Segurança Alimentar e Nutricional: 75 a $100 \%$ \\
total possível de pontos) & Insegurança Alimentar Leve: 50 a $74,9 \%$ \\
\hline Cobertura da ESF & Insegura Alimentar Moderada: 25 a $49,9 \%$ \\
& Alta: $>70 \%$ \\
& Média: $70 \%-50 \%$ \\
\hline
\end{tabular}

Fonte: ${ }^{9,10,16,18}$

O IDHM é um índice reconhecido internacionalmente e segue as três dimensões do desenvolvimento humano: longevidade, educação e renda. Tem longo uso nos estudos sociais, pois contempla outras dimensões, além da econômica. Por esse motivo, é considerado um indicador transparente e simplificado. ${ }^{15} \mathrm{O}$ índice varia de 0 a 1 , sendo o mais próximo de 1 , indicador de maior nível de desenvolvimento humano. Foram utilizados os dados do Atlas de Desenvolvimento Humano no Brasil, do Programa das Nações Unidas para o Desenvolvimento. ${ }^{10}$ 
A SAN é, ao mesmo tempo, uma diretriz e um princípio da PNAN, pois se trata de um elemento transversal em suas ações. Para esta associação, utilizou-se o estudo desenvolvido por Pereira, ${ }^{16}$ que, a partir da aplicação de um protocolo de indicadores, avaliou a condição de SAN em 57 municípios baianos, organizados segundo o porte populacional. O referido protocolo usou dados municipais secundários disponíveis em bases de acesso público e aferiu a SAN nas quatro dimensões - disponibilidade, acesso e consumo de alimentos e utilização biológica de nutrientes -, classificando a situação municipal em: SAN; Insegurança Alimentar Leve, Insegurança Alimentar Moderada e Insegurança Alimentar Grave. ${ }^{16,17}$

Ainda segundo a PNAN, a Atenção Básica à Saúde é o lócus privilegiado para o desenvolvimento das ações. Nesse âmbito de atenção à saúde, a ESF é compreendida como uma estratégia de reorientação do modelo de atenção, que enfatiza o trabalho comunitário e longitudinal. Utilizou-se o porcentual de cobertura populacional da ESF, com dados disponíveis no portal do Departamento de Atenção Básica, ${ }^{18}$ apresentado em diversos estudos como indicador para avaliação da atenção básica. ${ }^{12,19}$ A estimativa de cobertura populacional de equipes de saúde da família no território é obtida pelo cálculo: no de ESF x 3.450/População IBGE (Instituto Brasileiro de Geografia e Estatística), com limitador de cobertura de $100 \%$.

A análise dos dados foi realizada por meio da versão 15.0 do software StatisticalPackage for Social Science for Windows (SPSS), através de uma análise exploratória ou descritiva das principais variáveis do estudo, que tiveram natureza categórica ou numérica. Foi aplicado nas análises bivariadas o teste Qui-Quadrado, para verificação de diferenças de proporções; e o Teste T de Student, para avaliar as possíveis associações entre a existência do programa e o perfil nutricional de crianças menores de 5 anos. Foram considerados como resultados estatisticamente significativos os valores de $\mathrm{p}<0,05$.

\section{Resultados}

\section{O desenho da Política Nacional de Alimentação e Nutrição}

A PNAN surge após um histórico de iniciativas na área alimentar e nutricional, passando a contemplar programas que já eram desenvolvidos, a exemplo do Sistema de Vigilância Alimentar e Nutricional, apresentados em novos formatos, além de incluir uma série de novas iniciativas demandadas pelo perfil epidemiológico e nutricional da população, com vistas à articulação e à ampliação do escopo de atuação da ação pública na área.

A partir da política, foram identificados quatro eixos de ação da PNAN, os quais englobam uma série de programas e ações. O primeiro eixo, o de Vigilância Alimentar e Nutricional, contempla 
ações que têm como objetivo garantir informações necessárias ao acompanhamento do estado nutricional e alimentar da população, como incentivos à pesquisa e aos inquéritos populacionais. Para essa linha, o programa desenvolvido pelo município é o Sistema de Vigilância Alimentar e Nutricional (SISVAN), aliado ao Programa Bolsa Família (PBF), que prevê condicionalidades no setor saúde, entre elas, o acompanhamento do estado nutricional de crianças e mulheres beneficiárias.

O segundo eixo é o da Promoção da Saúde, que inclui iniciativas de cunho educativo, como os guias alimentares e as ações de Educação Alimentar e Nutricional (EAN). Além desses, há o programa Peso Saudável, uma iniciativa voltada para os profissionais do Ministério da Saúde e para a ação da Agência Nacional de Vigilância Sanitária (ANVISA) - prevista na política, mas de atuação mais ampla nos diversos setores. Ao nível municipal, destacam-se as ações de EAN e o Programa Saúde na Escola (PSE), que visa à articulação entre Escola e Rede Básica de Saúde, por meio de uma série de ações, entre as quais se sobressaem as na área alimentar e nutricional, orientada pela PNAN.

O terceiro eixo é o de Prevenção e Controle de Agravos Nutricionais, que inclui os programas de suplementação e de fortificação de ferro e ácido fólico, de suplementação de vitamina A, de polivitamínico (NutriSUS) e iodação do sal. Esses programas são desenvolvidos em âmbito municipal. Nesse eixo estão incluídas também as Linhas de Cuidado, voltadas para a organização da rede de atenção aos casos de desnutrição e de doenças crônicas, além da vigilância nutricional nos casos de beribéri. E, mais recentemente, emergem ações voltadas para indivíduos com necessidades alimentares especiais, visando garantir assistência a esse público. Esta também é uma iniciativa desenvolvida em âmbito local.

O quarto eixo - o da Pesquisa, Inovação e Conhecimento - contempla incentivos à pesquisa aos Centros Colaboradores de Alimentação e Nutrição do Escolar (CECANE) e a Rede Nutri, uma rede digital de informações em alimentação e nutrição. Este escopo de ações, apesar de contar com colaborações municipais, não contempla atividades de responsabilidade da gestão municipal.

Conforme a política, o âmbito estadual deve prestar assessoria técnica e apoio institucional aos municípios e às regionais de saúde no processo de gestão, planejamento, execução, monitoramento e avaliação de programas e ações de alimentação e nutrição, além de realizar capacitação de recursos humanos e destinar recursos estaduais para compor o financiamento tripartite com vistas à implementação da política no estado.

A PNAN foi implantada no estado simultaneamente ao âmbito nacional. Identificou-se, a partir da entrevista realizada, que a gestão estadual assume, especialmente, o monitoramento de programas através de indicadores de cobertura e de metas, com incentivo a sua implementação.

[...] a PNAN em si está implantada e sendo implementada com o passar do tempo, sendo fortalecida e melhorada. Agora, o que a gente percebe é que a gente ainda precisa andar muito [...] O programa de Vitamina A, por exemplo, há distribuição de capsulas, mas há uma lacuna ainda com relação ao sistema de informação. Muitos 
não registram as informações no sistema. Então a gente precisa qualificar e quantificar ainda os registros no sistema de informação (Gestora estadual de Saúde, 2015).

\section{Implantação dos programas em âmbito local}

Sobre o desenvolvimento de ações no âmbito municipal, $80 \%$ (44) dos gestores/responsáveis afirmaram que a política estava implantada no município, e os demais $20 \%$ (11) não apresentaram a PNAN estabelecida, ainda que fizessem menção à existência de programas específicos. Dispunham de responsável técnico pelas ações, 96,4\% (53) dos municípios, sendo as principais formações: enfermeiro (29), nutricionista (19) ou outro profissional de nível superior (4). Não dispunham, dois dos municípios.

As ações mais encontradas nos municípios pesquisados foram o PSE, o programa nacional de suplementação de ferro e ácido fólico, a suplementação de vitamina A e o acompanhamento das condicionalidades de saúde do programa Bolsa Família. Iniciativas mais recentes, e que envolvem uma atenção em rede, como a Atenção Nutricional à Desnutrição Infantil (ANDI), a linha de cuidado das doenças crônicas e atenção às necessidades alimentares especiais, tiveram menos citações. As Ações de EAN foram indicadas como existentes em mais de $80 \%$ dos municípios pesquisados. Contudo, o presente estudo não investigou como se constituíram e em que espaços aconteceram essas ações. Atividades de educação permanentes, como a Estratégia Amamenta e Alimenta Brasil, foram pouco presentes, em apenas 14,5\% (8) da amostra.

Os resultados sobre a cobertura dos programas por unidades de saúde indicaram uma boa distribuição das ações pelo território, desenvolvidas em mais de $50 \%$ das unidades para todos os programas.

Sobre a aplicação de recursos municipais no desenvolvimento das atividades, todos os municípios relataram o uso de recursos próprios em pelo menos uma das ações, variando conforme a ação. O PSE e o programa de suplementação de ferro e ácido fólico são os programas que receberam mais investimentos do município, segundo os entrevistados. Considerando apenas os municípios que têm os programas, o planejamento foi relatado em, aproximadamente, $50 \%$ dos municípios. Já a avaliação foi menos referida.

Quando consideradas todas as variáveis simultaneamente para avaliar o grau de implantação dos programas, observou-se que iniciativas de maior longevidade alcançaram maior porcentual no grau de implantação satisfatório nos municípios, principalmente quando comparadas às iniciativas mais recentes (Tabela 1). A implantação completa ainda é diminuta, estando os programas Bolsa Família e PSE com maiores porcentuais. A estratégia Alimenta e Amamenta Brasil e as Linhas de Cuidado para doenças crônicas foram as de menor porcentual de implantação completa, com $5,44 \%$ (3) de presença dessas atividades. 


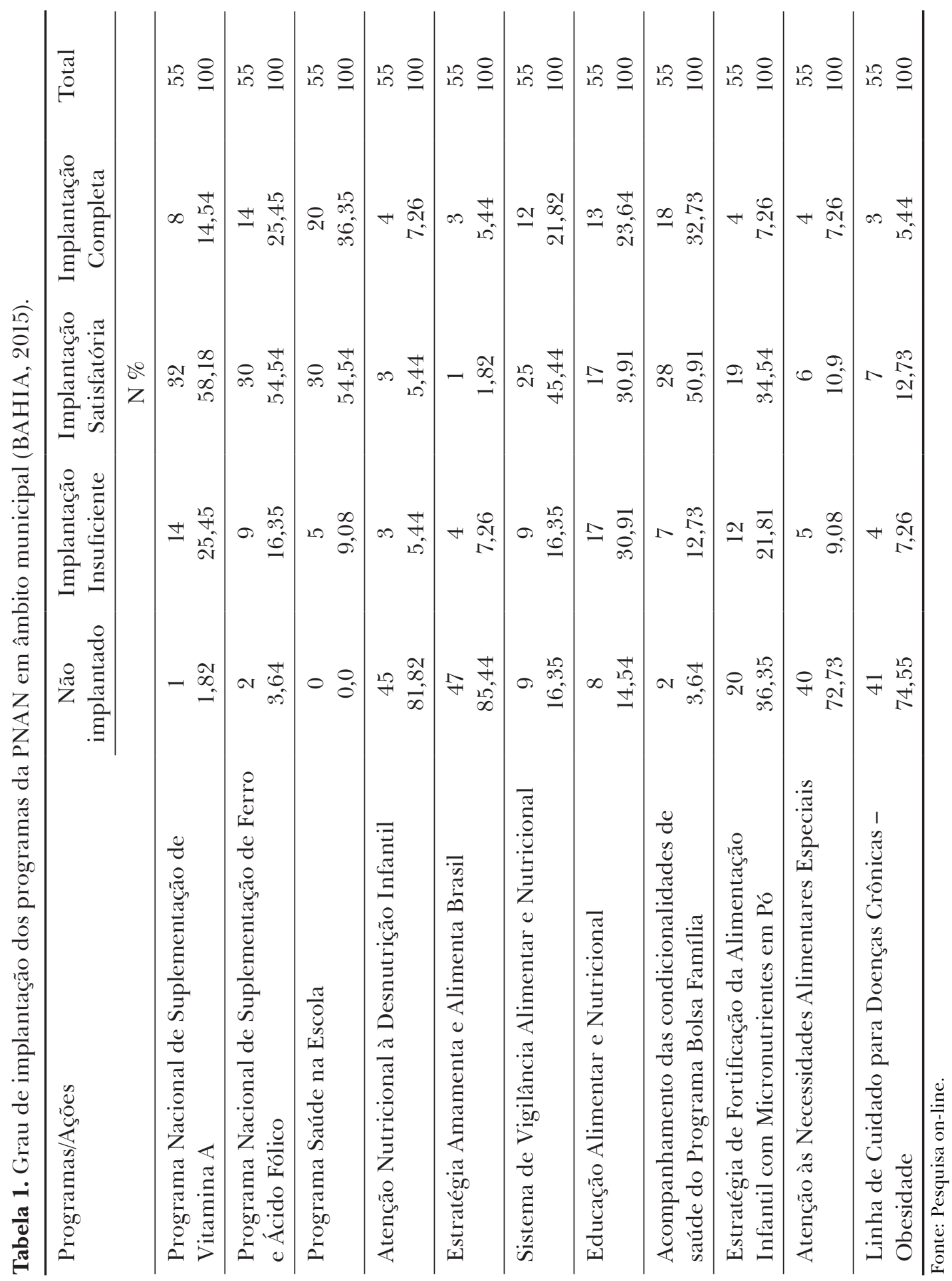


Programas e associaçã̃o com indicadores sociodemográficos

Dos municípios investigados, 63,3\% (35) apresentam IDHM baixo, seguido por 34,5\% (19) com IDHM médio e 1,8\% (1) com IDHM alto. ${ }^{10}$ Segundo o porte populacional, 50,9\% (29) foram classificados como urbano pequeno I e 38,2\% (22) como urbano pequeno II e. Outros dois foram identificados como de médio porte e quatro, de grande porte. ${ }^{9}$

Sobre a cobertura populacional estimada da ESF, espaço onde se desenvolvem a maior parte dos programas da PNAN, 87,3\% (50) dos municípios apresentaram cobertura superior a 70\%, classificada como alta. Os demais 9,1\% (5), cobertura média e 3,6\%, (2) baixa. ${ }^{18}$ Em relação à situação de Insegurança Alimentar e Nutricional (ISAN) nos municípios, 74,5\% (43) indicaram ISAN Moderada; 20,0\% (11), ISAN Leve; e 5,5\% (3), ISAN Grave. ${ }^{16}$ Nenhum dos municípios apresentou situação de Segurança Alimentar e Nutricional.

O IDHM municipal alto apontou associação significativa com os programas NutriSUS e de atenção às necessidades alimentares especiais $(\mathrm{p}=0,013 \mathrm{e} \mathrm{p}=0,026)$ (Tabela 2). A presença do programa NutriSUS, do acompanhamento da condicionalidade de saúde do PBF e da Atenção às Necessidades Alimentares Especiais apresentou associação expressiva em municípios com maior cobertura da Estratégia de Saúde da Família ( $p=0,032 ; p=0,029 ; p=0,000)$. 
Tabela 2. Associação entre programas da PNAN e indicadores sociais. Bahia, 2015. (continua)

\begin{tabular}{|c|c|c|c|c|c|c|c|c|c|c|}
\hline \multirow{5}{*}{$\begin{array}{l}\text { Variáveis } \\
\text { Política/ } \\
\text { Programas }\end{array}$} & \multicolumn{5}{|c|}{ IDHM } & \multicolumn{5}{|c|}{ Cobertura ESF } \\
\hline & \multicolumn{2}{|c|}{ IDHM alto } & \multicolumn{2}{|c|}{ IDHM baixo } & \multirow{4}{*}{$\mathrm{p}$} & \multicolumn{2}{|c|}{$\begin{array}{c}\text { Cobertura } \\
\geq 70 \%\end{array}$} & \multicolumn{2}{|c|}{$\begin{array}{c}\text { Cobertura } \\
<70 \%\end{array}$} & \multirow{4}{*}{$\mathrm{p}$} \\
\hline & Sim & Não & Sim & Não & & Sim & Não & Sim & Não & \\
\hline & $\mathrm{N}$ & $\mathrm{N}$ & $\mathrm{N}$ & $\mathrm{N}$ & & $\mathrm{N}$ & $\mathrm{N}$ & $\mathrm{N}$ & $\mathrm{N}$ & \\
\hline & $(\%)$ & $\%$ & $\%$ & $\%$ & & $\%$ & $\%$ & $\%$ & $\%$ & \\
\hline \multirow[t]{2}{*}{ PNAN } & 18 & 2 & 26 & 9 & 0,161 & 37 & 11 & 7 & 0 & 0,157 \\
\hline & 40,9 & 18,2 & 59,1 & 81,8 & & 84,1 & 100 & 15,9 & 0 & \\
\hline Ferro/Áci-do & 20 & 0 & 33 & 2 & 0,276 & 46 & 2 & 7 & 0 & 1 \\
\hline Fólico & 37,7 &, 0 & 66,3 & 100 & & 86,8 & 100 & 13,2 & 0 & \\
\hline \multirow[t]{2}{*}{ VIT A } & 20 & 0 & 34 & 1 & 0,446 & 47 & 1 & 7 &, 0 & 0,700 \\
\hline & 37 &, 0 & 63 & 100 & & 87 & 100 & 13 & & \\
\hline \multirow[t]{2}{*}{ ANDI } & 4 & 16 & 7 & 27 & 0,747 & 9 & 38 & 2 & 5 & 2 \\
\hline & 36,4 & 37,2 & 63,8 & 62,8 & & 81,8 & 88,4 & 18,2 & 11,6 & \\
\hline \multirow[t]{2}{*}{ EAAB } & 4 & 15 & 4 & 31 & 0,263 & 5 & 42 & 3 & 4 & 0,073 \\
\hline & 50 & 32,6 & 50 & 67,4 & & 62,5 & 91,3 & 37,5 & 8,7 & \\
\hline \multirow[t]{2}{*}{ SISVAN } & 18 & 2 & 28 & 7 & 0,335 & 39 & 9 & 7 & 0 & 1 \\
\hline & 39,1 & 22,2 & 60,9 & 77,8 & & 84,8 & 100 & 15,2 & 0 & \\
\hline \multirow[t]{2}{*}{ EAN } & 19 & 1 & 27 & 7 & 0,220 & 39 & 7 & 8 & 0 & 2 \\
\hline & 41,3 & 12,5 & 58,7 & 87,5 & & 84,8 & 15,2 & 100 & 0 & \\
\hline \multirow[t]{2}{*}{ PBF } & 18 & 1 & 35 & 0 & 0,163 & 47 & 1 & 6 & 0 & $0,029 *$ \\
\hline & 34 & 100 & 66 & 0 & & 88,7 & 100 & 11,3 & 0 & \\
\hline \multirow[t]{2}{*}{ NutriSUS } & 17 & 3 & 18 & 17 & $0,013^{*}$ & 28 & 20 & 7 & 0 & $0,032 *$ \\
\hline & 48,6 & 15 & 51,4 & 85 & & 80 & 100 & 20 & 0 & \\
\hline \multirow[t]{2}{*}{ ANAE } & 9 & 10 & 6 & 29 & $0,026^{*}$ & 10 & 38 & 5 & 1 & 0,00 \\
\hline & 60 & 25,6 & 40 & 74,4 & & 66,7 & 97,4 & 33,3 & 2,6 & $*$ \\
\hline \multirow[t]{2}{*}{ Lc Obesidade } & 6 & 14 & 8 & 27 & 1 & 11 & 37 & 3 & 4 & 0,258 \\
\hline & 42,9 & 34,1 & 57,1 & 65,9 & & 78,6 & 90,2 & 21,4 & 9,8 & \\
\hline
\end{tabular}


Tabela 2 (continuação)

\begin{tabular}{|c|c|c|c|c|c|c|c|c|c|c|}
\hline \multirow[t]{2}{*}{ Variáveis } & \multicolumn{5}{|c|}{ Situação de ISAN } & \multicolumn{5}{|c|}{ Porte Populacional } \\
\hline & \multicolumn{2}{|c|}{ ISAN Leve } & \multicolumn{2}{|c|}{$\begin{array}{c}\text { ISAN } \\
\text { Moderada Ou } \\
\text { Grave }\end{array}$} & \multirow{4}{*}{$\mathrm{p}$} & \multicolumn{2}{|c|}{$>100.000$ hab. } & \multicolumn{2}{|c|}{$<100.000$ hab. } & \\
\hline \multirow{3}{*}{$\begin{array}{l}\text { Política/ } \\
\text { Programas }\end{array}$} & Sim & Não & Sim & Não & & Sim & Não & Sim & Não & p \\
\hline & $\mathrm{N}$ & $\mathrm{N}$ & $\mathrm{N}$ & $\mathrm{N}$ & & $\mathrm{N}$ & $\mathrm{N}$ & $\mathrm{N}$ & $\mathrm{N}$ & \\
\hline & $\%$ & $\%$ & $\%$ & $\%$ & & $\%$ & $\%$ & $\%$ & $\%$ & \\
\hline \multirow[t]{2}{*}{ PNAN } & 9 & 2 & 35 & 9 & 0,666 & & & & & \\
\hline & 20,5 & 18,2 & 79,5 & 81,9 & & & & & & \\
\hline \multirow{2}{*}{$\begin{array}{l}\text { Ferro/Áci-do } \\
\text { Fólico }\end{array}$} & 11 & 0 & 42 & 2 & 0,471 & 4 & 0 & 49 & 2 & 0,687 \\
\hline & 20,8 & 0 & 79,2 & 100 & & 7,5 &, 0 & 92,5 & 100 & \\
\hline \multirow[t]{2}{*}{ VIT A } & 11 & 0 & 43 & 1 & 0,614 & 4 & 0 & 50 & 1 & 0,777 \\
\hline & 20,4 & , 0 & 79,6 & 100 & & 7,4 & ,0 & 92,6 & 100 & \\
\hline \multirow[t]{2}{*}{ ANDI } & 6 & 5 & 5 & 38 & $0,006^{*}$ & 2 & 2 & 9 & 42 & 0,119 \\
\hline & 54,5 & 11,6 & 45,5 & 88,4 & & 18,2 & 4,5 & 81,8 & 95,5 & \\
\hline \multirow[t]{2}{*}{ EAAB } & 3 & 8 & 5 & 38 & 0,372 & 2 & 2 & 6 & 44 & 0,111 \\
\hline & 37,5 & 17,4 & 62,5 & 82,6 & & 25 & 4,3 & 75 & 95,7 & \\
\hline \multirow[t]{2}{*}{ SISVAN } & 10 & 1 & 36 & 8 & 0,466 & 4 & 0 & 42 & 9 & 0,358 \\
\hline & 21,7 & 11,1 & 78,3 & 88,9 & & 8,7 &, 0 & 91,3 & 100 & \\
\hline \multirow[t]{2}{*}{ EAN } & 11 & 0 & 35 & 8 & 0,261 & 4 & 0 & 42 & 8 & 0,656 \\
\hline & 23,9 & 0 & 76,1 & 100 & & 8,7 &, 0 & 91,3 & 100 & \\
\hline \multirow[t]{2}{*}{ PBF } & 11 & 0 & 42 & 1 & 0,771 & 6 & 0 & 50 & 1 & 0,001 \\
\hline & 20,8 & 0 & 79,2 & 100 & & 5,7 &, 0 & 94,3 & 100 & $*$ \\
\hline \multirow[t]{2}{*}{ NutriSUS } & 8 & 3 & 27 & 17 & 1 & 4 & 0 & 31 & 20 & 0,116 \\
\hline & 22,9 & 15 & 77,1 & 85 & & 11,4 & ,0 & 88,6 & 100 & \\
\hline \multirow[t]{2}{*}{ ANAE } & 5 & 6 & 10 & 33 & 0,296 & 3 & 0 & 12 & 39 & 0,000 \\
\hline & 33,3 & 15,4 & 66,7 & 84,6 & & 20,0 &, 0 & 80 & 100 & $*$ \\
\hline \multirow[t]{2}{*}{ Lc Obesidade } & 4 & 7 & 10 & 34 & 0,353 & 2 & 2 & 12 & 39 & 0,242 \\
\hline & 28,6 & 17,1 & 71,4 & 82,9 & & 14,3 & 4,9 & 85,7 & 95,1 & \\
\hline
\end{tabular}

Fonte: Pesquisa on-line..$^{9,10,16,18}$

\section{Legenda:}

Ferro/Ácido Fólico: Programa Nacional de Ferro e Ácido Fólico; VIT A: Programa Nacional de Suplementação de Vitamina A; ANDI: Atenção Nutricional a Desnutrição Infantil; EAAB: Estratégia Amamenta e Alimenta Brasil; SISVAN: Sistema de Vigilância Alimentar e Nutricional; EAN: Educação Alimentar e Nutricional; PBF: Programa Bolsa Família; NutriSUS: Estratégia de fortificação da alimentação infantil com micronutrientes em pó; ANAE: Atenção às Necessidades Alimentares Especiais; Lc Obesidade: Linha de Cuidado para pessoas com obesidade 
Municípios com ISAN Grave apresentaram associação estatisticamente significante com a baixa presença do programa de Atenção Nutricional à Desnutrição Infantil $(p=0,006)$. As outras variáveis não mostraram associações estatisticamente significantes. Em relação ao PSE, não foi possível verificar associação, pois esteve presente em 100\% dos municípios.

Para o Programa Nacional de Suplementação de Ferro, observou-se que os municípios que dispõem de tal ação apresentaram maior porcentual médio de crianças com magreza (Excesso de peso $p=0,435$; Magreza $p=0,000$; Eutrofia $p=0,795$ ), assim como foi encontrado nos municípios onde não são desenvolvidas ações de Educação Alimentar e Nutricional (Excesso de peso p= 0,382; Magreza $\mathrm{p}=0,031$; Eutrofia $\mathrm{p}=0,657)$.

Municípios que não realizaram o acompanhamento da condicionalidade de saúde do programa Bolsa Família apresentaram maior porcentual médio de magreza (Excesso de peso p=0,575; Magreza $p=0,006$; Eutrofia $p=0,406$ ); enquanto municípios em que não havia a Estratégia de Fortificação NutriSUS apresentaram maior porcentual médio de excesso de peso (Excesso de peso $\mathrm{p}=0,034$; Magreza $\mathrm{p}=0,544$; Eutrofia $\mathrm{p}=0,301$ ).

\section{Discussão}

A caracterização da PNAN e de suas ações revelam o quanto a política é ampla e dinâmica, o que a torna complexa e de difícil implantação. Esta avaliação tem limitações de amostragem, particularidade desse tipo de investigação, pois envolve a disponibilidade de gestores na geração das informações. No entanto, apresenta resultados significativos e relevantes para área.

O levantamento das iniciativas propostas pela política e o da implantação de programas no âmbito local, esfera na qual a ação chega ou não ao seu destino, o usuário, revelaram discrepâncias consideráveis. Propor uma atuação individualizada em distintas frentes é o diferencial da PNAN, após uma série de experiências de baixa efetividade e seletivas na área, anteriores a esta perspectiva. Contudo, o distanciamento entre a gestão federal e a local ainda é um entrave na esfera pública.

Os programas de maior tempo de existência apresentaram maior porcentual de implantação satisfatória nos municípios com relação às ações/programas mais recentes, o que indica um movimento positivo de consolidação das ações. No entanto, considerando este mesmo critério, ações de maior tempo deveriam indicar um maior porcentual de implantação completa, o que não foi observado. Por outro lado, tendo em vista a transição nutricional e o foco recente na promoção da saúde e da alimentação saudável, este resultado pode sugerir dificuldades para a introdução de estratégias diferenciadas. 
Estudos sobre o modelo de atenção à saúde vigente fazem menção a uma crise no sistema de saúde, caracterizado por um desencontro entre as demandas epidemiológicas marcadas por condições crônicas e uma organização voltada a responder condições agudizadas.$^{20}$ Isso parece estar se refletindo também nas ações de alimentação e nutrição, que têm priorizado ações para condições já instaladas de distúrbios nutricionais em detrimento daquelas que promovam um estilo de vida e alimentação saudável.

Levando em conta os elementos utilizados para classificar a implantação, o planejamento e a avaliação apresentaram-se como entraves, como práticas pouco incorporadas. No Brasil, tem-se que o planejamento e a avaliação ainda são escassos na administração pública. O planejamento, quando acontece, ainda é visto como etapa burocrática distante da realidade, ${ }^{21} \mathrm{e}$ a avaliação, como instrumento de apoio à gestão na melhoria do processo decisório, é pouco utilizada. Segundo destacado por Henrique et al., ${ }^{22}$ há uma ausência de uma "cultura de avaliação", o que pode comprometer os resultados esperados na implementação dos programas.

Propostas de menor tempo de criação tiveram altos valores de não implantação, indicando baixa adesão às novas iniciativas. O esforço no âmbito da gestão federal é basilar no desenvolvimento das ações da PNAN e nas de alimentação e nutrição ao longo dos anos, mas não garante que as ações sejam implementadas em nível local, pois este é dotado de autonomia na administração de programas. Arretche ${ }^{23}$ problematiza a interpretação dada à gestão descentralizada no Brasil, apontada enquanto forma democrática, que viabilizaria a concretização de ideais progressistas, como equidade, justiça social, redução do clientelismo e aumento do controle social sobre o Estado. Não nega que tais objetivos possam ser alcançados, mas debate que várias das expectativas que repousaram sobre esse conjunto de associações positivas não são um resultado necessário e automático da descentralização.

Não cabe ao escopo deste estudo analisar a descentralização, no entanto é possível contribuir com a discussão ao detectar o distanciamento entre uma proposta central de política e sua expressão em instâncias locais - onde são executadas -, a exemplo das linhas de cuidado para atenção aos casos de desnutrição e obesidade. E quando se trata de linhas de cuidado, outras demandas emergem, como o reduzido uso dessas estratégias nos serviços de saúde e a dificuldade de articulação da rede em fluxo contínuo de atenção ao usuário. ${ }^{24,25}$

A associação positiva encontrada entre maior IDHM e maior porcentual médio de excesso de peso, com a existência dos programas NutriSUS e de Atenção às Necessidades Alimentares Especiais nos municípios, pode estar relacionada a uma melhor condição econômica dos municípios, na medida em que são ações que demandam investimento local. O NutriSUS prevê a criação de creches em tempo integral, bem como ações de atenção às necessidades, prevê a compra ou o financiamento de alimentos para públicos especiais, a exemplo dos portadores de intolerâncias alimentares. 
Houve também a maior presença do acompanhamento da condicionalidade de saúde do programa Bolsa Família em municípios com maior cobertura da ESF. Nesse sentido, é possível considerar que a atenção básica é o espaço onde esta ação acontece. Levando em conta a prerrogativa de os municípios responsabilizarem-se em ofertar os serviços relativos às condicionalidades, as diferentes capacidades administrativas municipais podem repercurtir diferentes graus da assistência ofertada, ${ }^{26}$ bem como o atendimento aos usuários do programa pressupõe a organização da Atenção Básica para garantir a oferta de serviços, o que pode acontecer em maior ou menor proporção. ${ }^{27}$

A associação entre a baixa presença do Programa de Atenção Nutricional à Desnutrição Infantil e os municípios com ISAN grave pode indicar um baixo investimento em políticas sociais e/ou incapacidade econômica, o que tem repercutido na situação de ISAN dos municípios, bem como na própria prevalência de desnutrição entre crianças, indicador e resultado da ISAN. Tem-se que a medida de Segurança Alimentar esteve diretamente relacionada com as condições socioeconômicas das famílias. ${ }^{28,29}$ Vieira et al., ${ }^{30}$ a partir de estudo realizado em São Paulo, afirmaram que categorias de insegurança alimentar foram consideradas potenciais determinantes da desnutrição infantil.

O Programa Nacional de Suplementação de Ferro esteve relacionado ao maior porcentual médio de crianças com magreza. Ao contrário do que possa parecer, tal resultado pode estar associado a maior implantação do programa em municípios onde há maior vulnerabilidade social. Em estudo específico, a anemia e a deficiência de ferro apresentaram correlação com uma série de determinantes sociais, ${ }^{31}$ que se relacionam a baixos índices de desenvolvimento infantil, a exemplo da magreza em crianças.

Já sobre as ações de Educação Alimentar e Nutricional, essa associação foi contrária, estando menos presente quanto maior for o porcentual médio de crianças com magreza. Destaca-se que a EAN é um meio com vistas ao alcance da Segurança Alimentar e Nutricional, abarcando ações que visam reduzir as diversas manifestações de insegurança alimentar. ${ }^{32}$ Nesse sentido, a ausência dessas ações, junto a outros determinantes, pode estar relacionada com o maior porcentual de crianças com magreza.

Considerando a experiência brasileira de descentralização, a ausência de indução e de incentivos da esfera federal pode ser um fator relacionado ao insucesso das ações a serem implantadas. Pelos resultados obtidos neste estudo, e considerando os municípios participantes da pesquisa, permanecem sendo priorizadas as ações de controle de agravos em função de ações que visam à intervenção nos fatores causais e relacionados aos agravos nutricionais. Deste modo, é desafio desenvolver, fomentar e articular ações para cuidado, em um cenário de extrema desigualdade social. 


\section{Conclusão}

A PNAN é uma política pioneira e resultante de experiências exitosas e não exitosas anteriores a ela, assim como de mobilizações que levantaram a bandeira de ampliação de sua perspectiva. Assumiu assim papel estratégico no setor de saúde, levando em conta o perfil nutricional da população e almejando a segurança alimentar e nutricional dos brasileiros, por meio da atuação em diversas frentes, que exigiram o desenvolvimento de programas com diversas abordagens. Essa amplitude enriquece a política, mas aumenta também sua complexidade, ao considerar a estrutura (física, econômica e de recursos humanos) necessária ao seu desenvolvimento.

Tal complexidade é pouco explorada em estudos na área. Ao levantar fatores que influem na implantação e desenham um panorama da política no estado, colabora-se para uma maior compreensão de como a PNAN está desenhada para o usuário no âmbito municipal. Mais que isso, os resultados aqui discutidos revelam avanços e limites importantes à implantação de ações da PNAN. Entre os limites estão a diversificação das ações em função da reprodução de antigas estratégias de cuidado, modificações nos formatos tradicionais de trabalho em saúde para inclusão de novas propostas, o baixo estímulo a iniciativas mais recentes, gestão orientada aos problemas com planejamento e avaliação, financiamento e institucionalização da área alimentar e nutricional na atenção básica. Destaca-se enquanto facilitadores o estímulo e investimento federal das ações, por transferência fundo a fundo e expansão da atenção básica à saúde.

A determinação social dos agravos nutricionais é reafirmada ao verificar relação entre indicadores e a existência dos programas. Garantir a efetivação da implantação de todos os programas da PNAN é fortalecer direitos sociais. Levando em conta esses elementos, devem ser planejadas estratégias de difusão de iniciativas mais recentes da política, em especial as que propõem formatos não tradicionais de trabalho, a exemplo das linhas de cuidado, ações de Educação Alimentar e Nutricional e formação profissional em redes, e repensados os limites de ações já implantadas há mais tempo.

Observou-se lacunas na produção de conhecimentos do campo empírico sobre a implantação de programas da PNAN, com isto houve dificuldade de comparação com resultados semelhantes. O presente trabalho abre-se, no entanto, para uma série de novas demandas de pesquisa em relação à política, indicando vazios teóricos que merecem novas investigações.

\section{Agradecimento}

Agradecemos a Jerusa da Mota Santana, pelas contribuições nas análises estatísticas. 


\section{Colaboradores}

LMS Souza e SMC Santos participaram de todas as etapas, desde a concepção do estudo até a revisão da versão final do artigo.

Conflito de Interesses: Os autores declaram não haver conflito de interesses.

\section{Referências}

1. Brasil. Portaria No 710, de 10 de junho de 1999. Aprova a Política Nacional de Alimentação e Nutrição. Diário Oficial da União 15 jun. 1999; Seção 1.

2. Brasil. Ministério da Saúde. Política Nacional de Alimentação e Nutrição. Brasília: Ministério da Saúde; 2012.

3. Pinheiro ARO, Carvalho DBB. Estado e mercado: adversários ou aliados no processo de implementação da Política Nacional de Alimentação e Nutrição? Elementos para um debate sobre medidas de regulamentação. Saúde Soc. 2008; 17(2):170-183.

4. Escoda MSQ. Para a crítica da transição nutricional. Ciênc Saúde Coletiva 2002; 7(2):219-226.

5. Coutinho JG, Gentil PC, Toral N. A desnutrição e obesidade no Brasil: o enfrentamento com base na agenda única da nutrição. Cad Saúde Pública 2008; 24(2):332-340.

6. Arretche MTS. Uma contribuição para fazermos avaliações menos ingênuas. In: Barreira MCRN, Carvalho MCB, organizadoras. Tendências e perspectivas na avaliação de políticas e programas sociais. São Paulo: IEE/PUC-SP; 2001. p. 43-56.

7. Recine E, Vasconcellos AB. Políticas nacionais e o campo da Alimentação e Nutrição em Saúde Coletiva: cenário atual. Ciênc Saúde Coletiva, 2011; 16(1):73-79.

8. Instituto Brasileiro de Geografia e Estatísticas. Cidades [Internet]. Disponível em: http://cidades. ibge.gov.br/xtras/perfil.php?lang=\&codmun $=292740$

9. Instituto Brasileiro de Geografia e Estatística. Pesquisa de orçamentos familiares 2008-2009: antropometria e estado nutricional de crianças, adolescentes e adultos no Brasil. Rio de Janeiro: IBGE; 2010. 130 p.

10. Programa das Nações Unidas para o Desenvolvimento no Brasil. Atlas do desenvolvimento humano no Brasil [Internet]. Disponível em: http://www.atlasbrasil.org.br/2013/

11. Brasil. Sistema de Vigilância Alimentar e Nutricional. Acesso aos relatórios públicos do SISVAN. Disponível em: http://dabsistemas.saude.gov.br/sistemas/sisvan/login.php?acesso_negado=true

12. Henrique F, Calvo MCM. Avaliação do Programa Saúde da Família nos municípios do Estado de Santa Catarina, Brasil. Cad Saúde Pública 2008; 24(4):809-819. 
13. Pimentel FC, Albuquerque PC, Martelli PJL, Souza WV, Acioli RML. Caracterização do processo de trabalho das equipes de saúde bucal em municípios de Pernambuco, Brasil, segundo porte populacional: da articulação comunitária à organização do atendimento clínico. Cad Saúde Pública 2012; 28(Supl.):S146-S157.

14. Instituto Brasileiro de Geografia e Estatística. Contagem da população 2007. Disponível em: http:// www.ibge.gov.br/home/estatistica/populacao/contagem2007/default.shtm

15. Guimarães JRS, Jannuzz PM. Indicadores sintéticos no processo de formulação e avaliação de políticas públicas: limites e legitimidades. XIV Encontro Nacional de Estudos Populacionais; 20-24 set. 2004; Caxambú- MG. Porto Alegre: ABEP; 2004. p. 1-19.

16. Pereira MHQ. Avaliação da segurança alimentar e nutricional: contribuições em âmbito municipal [dissertação]. Salvador: Universidade Federal da Bahia; 2014.

17. Panelli- Martins BE, Santos SMC, Assis AMO. Segurança alimentar e nutricional: desenvolvimento de indicadores e experimentação em um município da Bahia, Brasil. Rev Nutr. 2008; 21(Supl.):65s-81s.

18. Brasil. Ministério da Saúde. Histórico de cobertura da estratégia de saúde da família. Disponível em: http://dab.saude.gov.br/portaldab/historico_cobertura_sf.php

19. Silva NC, Garnelo L, Giovanella L. Extensão de cobertura ou reorganização da atenção básica? A trajetória do Programa de Saúde da Família de Manaus-AM. Saúde Soc. 2010; 19(3):592-604.

20. Brasil. Conselho Nacional de Secretários de Saúde. A Atenção Primária e as Redes de Atenção à Saúde. Brasília: CONASS; 2015. 127 p.

21. Oliveira JAP. Desafios do planejamento em políticas públicas: diferentes visões e práticas. RAP 2006; 40(1):273-88.

22. Henrique FCS, Lira PIC, Santos SMC, Andrade SLLS. Tendência do campo de avaliação de intervenções públicas de alimentação e nutrição em programas de pós-graduação no Brasil: 19802004. Cad Saúde Pública 2007; 23(12):2972-2981.

23. Arretche MTS. Mitos da descentralização: mais democracia e eficiência nas políticas públicas? Disponível em: http://www.anpocs.org.br/portal/publicacoes/rbcs_00_31/rbcs31_03.htm

24. Franco TB, Magalhães Júnior HM. Integralidade na assistência à saúde: a organização das linhas do cuidado. In: Brito JC. O trabalho em saúde: olhando e experienciando o SUS no cotidiano. 2 ed. São Paulo: Hucitec; 2004.

25. Mendes EV. As redes de atenção à saúde. Ciênc Saúde Coletiva 2010; 15(5):2297-2305.

26. Senna MCM, Burlandy L, Monnerat GL, Schottz, V, Magalhães R. Programa Bolsa Família: nova institucionalidade no campo da política social brasileira? Rev Katál 2007; 10(1):86-94.

27. Jaime PC, Silva ACF, Lima AMC, Bortolini GA. Ações de alimentação e nutrição na atenção básica: a experiência de organização no Governo Brasileiro. Rev Nutr. 2011; 24(6):809-824.

28. Vianna RPT, Segall-Corrêa AM. Insegurança alimentar das famílias residentes em municípios do interior do estado da Paraíba, Brasil. Rev Nutr. 2008; 21(Sup.):111s-122s. 
29. Barroso GS, Sichieri R, Salles Costa R. Fatores associados ao déficit nutricional em crianças residentes em uma área de prevalência elevada de insegurança alimentar. Rev Bras Epidemiol. 2008; 11(3):484-494.

30. Vieira VL, Souza JMP, Cervato-Mancuso A. M. Insegurança alimentar, vínculo mãe-filho edesnutrição infantil em área de altavulnerabilidade social. Rev Bras Saúde Matern Infant. 2010; 10(2):199-207.

31. Almeida ER, Carvalho AT, Nilson EAF, Coutinho JG, Ubarana JA. Avaliação participativa do Programa Nacional de Suplementação de Vitamina A em um município da Região Nordeste do Brasil. Cad Saúde Pública 2010; 26(5):949-960.

32. Brasil. Marco de referência de educação alimentar e nutricional para as políticas públicas. Brasília: MDS; Secretaria Nacional de Segurança Alimentar e Nutricional; 2012.

Recebido: 17/11/2016

Aceito: 29/1/2016 
